\title{
3 Ein Plädoyer für ein Bewusstsein für die Qualität und Bedeutsamkeit der Sprache auch im Prozess der Digitalisierung in der Pflege
}

\author{
Britta March
}

Gelingende Kommunikation ist die hohe Kunst der gekonnten Interaktion. Die Anforderungen an ernsthaft miteinander kommunizierende Akteure, die den Anspruch einer zielführenden oder lösungsorientierten Kommunikation haben, sind immer hoch. Sie steigen zudem stets - explizit in der Pflege- und Gesundheitswirtschaft. Hier wird der Fokus auf die Pflege gelegt. Die Gründe liegen in der Gesellschaft des langen Lebens selbst. Menschen leben lange, wollen und können mittlerweile immer älter werden. Der Preis dafür ist die Hilfe- und Pflegebedürftigkeit. Um diesen Veränderungen und Anforderungen adäquat begegnen zu können, wurden Gesetzgebungen reformiert. In der Umsetzung und Ausgestaltung der vielen Lebensbereiche ist professionelles Wissen und Handeln unentbehrlich und systemisches Denken erforderlich, und systematisches Forcieren erstrebenswerter Weiterentwicklungen höchst erwünscht. Das Ziel ist klar: Hilfebedürftigen Menschen nebst Angehörigen soll mit Beeinträchtigen bestmögliche Lebensqualität ermöglichte werden. Ein beiderseits berechtigter, aber hoher Anspruch, der gelingende Kommunikation voraussetzt, um Ziele, Bedarfe, Bedürfnisse, Erfordernisse und Optionen zu formulieren, die korrespondieren ... oder eben auch nicht. Und als wäre dies nicht herausfordernd genug, leben wir in dem modernen Zeitalter der digitalen Revolution, die auch vor der Pflegebrache keinen Halt macht. In der Erwartung, dass die Digitalisierung auch in der Pflegewelt Erleichterungen bringt, den Mangel an Pflegeexperten kompensieren hilft und Gutes bringt, wird sie glorifiziert. Was die Digitalisierung genau bringen soll, ist nur in Teilen formuliert oder definiert. Deshalb muss die Frage beantwortet werden: Wird sie überhaupt die positiven Erwartungen erfüllen können, ohne dass verstanden wird, welch zentrale Rolle die Qualität und Präzision der Sprache für 
gelingende Digitalisierung hat? Der bewusste Gebrauch der (Fach-)Sprache und der professionelle Dialog, der es erst ermöglicht, der bestehenden Komplexität adäquat zu begegnen, Anforderungen zu formulieren und Definitionen entstehen lässt, kann die Digitalisierungsprozesse zum Erfolg führen.

\section{Bedeutsamkeit der analogen Sprache - Präzises Sprechen und Sprachvermögen}

Die Bedeutungen der gesprochenen Sprache, des Gemeinten, des Mitgemeinten und der Interpretationsspielräume sind alles andere als trivial - insbesondere, wenn man sich immer wieder vergegenwärtigt: Man kann nicht nicht kommunizieren. (Watzlawick 1969). Spräche jemand aus „Ich verstehe die Pflegeversicherung nicht“, gäbe es mehrere Interpretationsmöglichkeiten, die, ohne einen weiteren Kontext zu kennen, richtig sein können und ohne Erklärung oder Nachfragen für gravierende Fehldeutungen und falsche Konsequenzen sorgen könnten. So wäre es möglich, anzunehmen, das Fachwissen des Sprechers reiche nicht aus und es erschließe sich der Inhalt deshalb nicht. Es könnte aber auch sein, dass er aufgrund seiner sozialpolitischen Überzeugung heraus die Konzeption der Pflegeversicherung im Gesamtgefüge der Sozialgesetzbücher infrage stelle und was, wenn der Grund einzig und allein die Unzulänglichkeit seiner Sprachkenntnisse wäre und ihm nur keine Übersetzung vorläge? Einen ähnlichen Interpretationsspielraum hinterließe man, drehte man sich auf eine Frage oder einen Satz zur Pflegeversicherung einfach um und ginge fort. Womit deutlich wird, dass es bei Weitem nicht nur die gesprochene Sprache ist, die zählt. Gleichzeitig wird deutlich, welche Bedeutung der Präzision der Formulierung, die Wortwahl und der Kontext zukommt, noch dazu, wenn ein Megatrend wie die Digitalisierung wirkt, die Transformation von analogen Prozessen läuft und eine adäquate Überführung vorausgesetzt wird - das ist mehr als eine einfache Übersetzung das wäre lediglich Automation.

Aber von welchem Sprachbewusstsein und -vermögen ist auszugehen und welche Wachheit gegenüber den unmittelbaren Zusammenhängen der Sprachqualität und dem, was in der Digitalisierung erfolgen muss, damit sie leisten kann, was erwartet wird, darf angenommen werden? Darf auf ein Desinteresse der Sprache gegenüber geschlossen werden, wenn im Land der Dichter und Denker das Jugendwortes des Jahres 2016 „fly sein“ und im Jahr davor „läuft bei dir“ öffentlichkeitswirksam gekürt wird. Floskeln, die unter Jugendlichen umgangssprachlich beliebt und gebräuchlich sein mögen. Aber was sagen sie aus? Mit einer Güte oder Sprachqualität, gepflegtem Ausdruck oder ähnlichen Ansprüchen an Inhalte, Haltung oder Kultur, hat diese Entwicklung konkret wenig zu tun und führt den Titel dieser sprachlichen Jahresauszeichnung auf geradem Weg ad absurdum. Vor der ständigen Veränderungsdynamik in einer jeden lebendigen Sprache seien die Augen nicht verschlossen. Bevölkerungen und Gesellschaften verändern sich - ob durch wirtschaftliche oder politische Gründe und das ist gut so. In Veränderungen liegen immer Chancen. Dass sich grundlegende Veränderungen in der Cesellschaft durch multikulturelle Entwicklungen auch auf die Sprache auswirken, kann wertvoll sein, wenn diese Dynamiken aufmerksam beobachtet und ihnen aktiv begegnet wird und die Prozesse systemisch begleitet werden. Eine Sprachkultur benötigt keiner zum Selbstzweck. Sie ist dann zentral bedeutsam und kann nur entstehen, wenn sie als Teil eines gesellschaftlichen Wertesystems 
verstanden wird. Sprache verleiht einer Gesellschaft - und damit auch Professionen dann eine qualifizierte Stimme, wenn sie bewusst gelebt und (weiter-)entwickelt wird. Nur mit dem ausdrücklichen Willen zu einer qualifizierten Ausdrucksweise und einem entsprechend reichen Wortschatz - transportieren sich die Inhalte in Varianz, Präzision und Qualität. Sprache muss bewusst als gesellschaftlich bedeutsamer Werteprozess verstanden und gelebt werden. Eine Verarmung der Sprache wird immer Konsequenzen auf gesellschaftliche Entwicklungen haben, (professionelle) Entwicklungen hemmen und Qualität multidimensional mindern.

\section{Gesprochener Wert: Sprache - Kultureller Wandel oder Verfall?}

Weshalb aber scheint sich das charakteristische und wertvolle Wesen der deutschen Sprache an vielen Stellen aufzulösen? Weshalb macht es den Anschein, dass sich viele Mitbürger in diesem Land - unabhängig ihrer Herkunft - keine Mühe mehr geben, die Landessprache, in der wir uns wundervoll auszudrücken vermögen, gerade wegen ihrer Komplexität und den sich dadurch eröffnenden Optionen, lernen zu wollen. In der subjektiven Wahrnehmung entsteht der Eindruck, dass die Besonderheiten in Güte, Varianz und Schönheit in Deutschland wenig anerkannt sind und an Bedeutung verlieren. In der U-Bahn hört man Jugendliche untereinander in einer Sprache sprechen, in der Artikel und Präpositionen eliminiert wurden, in der sich arabische, englische, türkische und deutsche Wörter mischen und in der vollständige Sätze keine Rolle zu spielen scheinen. WhatsApp, Snapchat, Twitter, Instagram - alle Massenmedien-Phänomene - tragen sicher zur generellen Verrohung der Sprache bei. Weshalb aber spielt die Bedeutung von Qualität einer vielfältigen und bedeutsamen Sprache in der öffentlichen Diskussion, in der Auseinandersetzung zur wertigen Weiterentwicklung des Gesundheitssystems, eine nur untergeordnete oder sogar gar keine Rolle? Bei der Anforderung und Diskussion um gelingende Integration oder Inklusion von Zuwanderern oder Flüchtlingen wird das Erlernen der Sprache an vorderste Stelle gestellt und zur absoluten Bedingung erklärt. Dies hat seine Berechtigung, da ohne die Möglichkeit des gegenseitigen Verstehens Fremdheit bestehen bleibt. Die Analogie zur systematischen Digitalisierung als neuer systemischer mehrdimensionaler Anforderung ist bestechend und es der Rückschluss auf die Bedingung, die Sprache im Gesamtgeschehen der Digitalisierung als Dimension des Verstehens in den Fokus zu rücken, richtig und berechtigt.

Die Sprache ist ein gesellschaftliches Elixier von hoher Bedeutsamkeit mit zentraler Referenz auf unser Selbst. Der Linguist und seit vielen Dekaden renommierter Sprachwissenschaftler Noam Chomsky stellt auch aktuell wieder die Frage nach dem, was Sprache ist und setzt den bemerkenswerten Aspekt unseres Selbst mit der Sprache gleich (Chomsky 2016, 38). Damit ist Sprache eines jeden human-immanente Identität, die gleichsam Verantwortung impliziert, bezieht man konsequent ein, was Sprache kann und bewirken soll(te) - sinnorientierter Gebraucht vorausgesetzt. Umso absurder die Beobachtung der Tendenz einer Sprache, um deren Bedeutung ausschließlich geschlossene Gruppen wissen. Bellinger weiß, dass es in die verkehrte Richtung geht, wenn Informationen mehr Wert erhalten als die Information selbst (Bellinger u. Krieger 2014, 33). Wenn das immanente Teilsystem ein anderes in Konkurrenz, nicht aber in der Qualität attackiert. 
„Sich um Verständlichkeit zu kümmern ist ebenso wichtig, wie sich um Fakten und Technologien zu kümmern. [...] (Es ist nicht die Frage, wer intelligenter werden muss, Kunde oder Produzent, Patient oder Pharmaindustrie.) Konzentrieren wir uns besser aufdas, was wirklich funktioniert: Verständlichkeit.“ (Bellinger u. Krieger 2014, 32ff.)

Eine unübliche Forderung explizit für das Gesundheitssystem, aber von einem Patienten, der spracherprobt und wissend ist, dass es nur über die Sprache läuft. Und weshalb fehlt die Debatte über Verantwortung einer Sprachqualität so gänzlich, die doch Basis aller Weiterentwicklung von Kultur und Gesellschaft ist, diese unmittelbar bedingt oder - schlimmer noch - bei Abwesenheit sogar verhindert? Und welche Folgen wird dies für die Gesellschaft der Zukunft haben, für die allgemeingültige Gesetze konzipiert sowie Ziele und Aufgaben formuliert werden müssen, auch in die, die dann in die digitale Übersetzung münden? Was, wenn das Verantwortungsgefühl für das Allgemeinwohl ein Auslaufmodell wird, weil das generelle Verständnis nicht mehr da sein kann und wir uns gesellschaftlich sprachlich eher ein postmodernes Babylon erschaffen und dies unkritisch zulassen?

Das Szenario eines drohenden Babylon, das unweigerlich das unbeherrschbare Chaos nach sich zöge, mag zu pessimistisch sein. Widmen wir uns konzentriert der Pflegebranche, der Sprache, die die Branche benötigt und den Erfordernissen, die explizit die Digitalisierung an Experten stellt oder stellen sollte. Denn die Abhängigkeit zwischen präziser Sprache und den Digitalisierungsprozessen und -zielen scheint mir weder ausreichend umfassend bewusst noch diskutiert. Daraus ergibt sich die Frage nach dem Stellenwert der Sprache. Sie muss gestellt werden und differenziert diskutiert und beantwortet werden - gerade im Prozess der fortschreitenden Digitalisierung, denn radikal formuliert kann die Digitalisierung im Anspruch großer entstehender zusammenhängend einwandfrei funktionierender Systeme nur mittels exakter Wortwahl, die Bedeutung klar generieren und den so formulierten Zielen gelingen. Digitalisierung macht sich nicht von selbst und setzt komplexes Wissen nicht allein in Computer- bzw. Programmiersprachen voraus. Diese geben qua Definition, in der Analogie zur beschriebenen Herausforderung an die menschliche gesprochene (ggf. auch geschriebene) Sprache, einen zentralen Gedanken wieder: Eine Voraussetzung dafür, dass der Computer die Erleichterungen oder Weiterentwicklungen der Prozesse überhaupt erbringen kann, ist der Mensch gefordert, die erforderliche Sprache präzise zu erlernen und zu beherrschen:

„Eine Programmiersprache ist eine künstliche Sprache zur Verständigung zwischen Mensch und Computer. Sie ist durch ihre Syntax (Syntax einer Programmiersprache) und Semantik (Semantik einer Programmiersprache) definiert. In einer Programmiersprache stellt man Verfahren zur Problemlösung in einer für den Computer,verständlichen Form dar." (Gabler Wirtschaftslexikon o.J.)

\section{Präzision im Sprechen und der Sprache - Die Basis aller Weiterentwicklung}

Die Voraussetzung ist exzellenter Umgang mit Wörtern und deren Bedeutung, die Inhalte schaffen oder die Erfordernisse konkret beschreiben. Diesen folgen dann digitale neue Prozessbeschreibungen. Es sei die Formulierung der These gewagt, dass 
die Qualität und Präzision der digitalen Prozesse umso höher wird, je ausführlicher, ggf. sogar virtuoser und damit gekonnter im Vorfeld sprachlich analog mit dem Problem oder Thema umgegangen wird. Da bereits aktuell angestoßene Digitalisierungsprozesse konkreten Zeitvorgaben unterliegen, stringentes Vorankommen erwartet wird und Deutschland im Pflegesektor nicht gerade Vorreiter dieser Entwicklungen ist, ist den Akteuren grundsätzlich zu raten, sich aktiv auf den Weg zu machen, weitere Entwicklungsprozesse zu initiieren. Dass es aktive, kluge und erfahrene Branchenkenner mit den richtigen Zielen gibt, die sich auf den Weg machen und mutig voran gehen, sei unbestritten. Dass dabei immer das Gesamtgefüge ausreichend beleuchtet und der Präzision der Sprache genügend Aufmerksamkeit gegeben wird, ist zumindest infrage zu stellen und anzuraten, falls nicht. Bei allem Bewusstsein für zügiges Vorankommen ist Zeit, die in präzise Definitionen und Ziele investiert wird, lohnenswert und erfolgsorientiert.

Die Erkenntnisse derer, die die Gesamtentwicklung effektiv befördern sollten, enttäuscht. Auch mit einem eHealth-Gesetz heißt es auf der Internetseite des Bundesministeriums für Wirtschaft lapidar:

„Die Digitalisierung ist in vollem Gange. Sie betrifft uns alle - und sorgt für einen tiefgreifenden Wandel in jedem Lebensbereich. Die digitale Transformation eröffnet dabei große Chancen für mehr Lebensqualität, revolutionäre Geschäftsmodelle und effıienteres Wirtschaften."(BMWI o.J.)

Das klingt einfach ... - bei der Sendung mit der Maus folgte an dieser Stelle: ... ist es aber nicht. Und was die sprachliche Qualität der Aussagekraft anbelangt nicht gerade preisverdächtig und mehr als wage. Was heißt „,voll in Gange“? Was bedeutet „,betrifft uns alle“? Welche Chancen werden wem eröffnet? Derartige Aussagen helfen vielleicht kosmetisch und sorgen oberflächlich für den Beweis, dass sich die Regierung kümmert. Da die ernstzunehmende Digitalisierung aber konkret Prozesse signifikant verändern, bestenfalls optimieren und den modernen Bedingungen entsprechen soll, wäre etwas mehr sprachliche Präzision oder Verweise zu Konkretem wünschenswert. Um dies aber zu können, müssen die hohen Erwartungen, die mit der Digitalisierung einhergehen, detailliert bekannt sein, sowie die Mechanismen der komplexen Bedingungen und Anforderungen gleichzeitig beachtet werden. Das ist alles andere als trivial. Und es reicht eben nicht, zu wissen, dass die Digitalisierung läuft, uns alle betrifft und Chancen eröffnet. Auch die folgende Erkenntnis, die der Bundesminister für Gesundheit, Hermann Gröhe, im Januar dieses Jahres in einem Interview äußerte, verweist ausschließlich auf Allgemeinplätze und fordert generell und unkonkret:

„Die Digitalisierung ist der mächtigste Treiber der Veränderung nicht nur in unserer Lebens-und Arbeitswelt, sondern auch im Gesundheitswesen. Sie birgt viele Chancen für mehr Lebensqualität und eine bessere Gesundheitsversorgung. Im Mittelpunkt stehen muss der Nutzen für die Versicherten und die Bedürfnisse der Patienten: hohe Versorgungsqualität, Teilhabe am medizinischen Fortschritt, Selbstbestimmung, Verständlichkeit und Sicherheit. Daran muss sich eine, digitale Gesundheitsagenda' ausrichten." (BMG 2017) 


\section{Synthese und Interdependenzen zwischen Sprache und Digitalisierung}

Und keiner spricht im Zusammenhang des Digitalisierungsprozesses generell - in der Pflege schon gar nicht - je von der Bedeutung der Kommunikation und der Sprache. Projekte zur Digitalisierung aber werden überall ins Leben gerufen, machen eine Art Karriere, auch in der Pflege. Konzeptionen werden entworfen, aber wird die Kommunikation dort ausreichend geführt, in der sich die Weichen für die Lösungen der Zukunft stellen? Kurzfristige Lösungen im nur kurzfristig sinnvoll erscheinenden „Klein-Klein“ sind nicht die, die in die erfolgreiche digitale Zukunft führen. Notwendig und anzustreben sind aber die „großen Lösungen“, die langfristig in die Zukunft führen, die - auf die Digitalisierung in der Pflege geschaut - systemisch und systematisch Veränderungen und Erneuerungen bringen! Einerseits braucht das System echte Verschlankung, massiven Service, der leicht zu generieren ist, andererseits unkomplizierte Vernetzungsoptionen aller Art um Sozialraumgestaltung tatsächlich umfassend ganzheitlich und sinnvoll planen und umsetzen zu können. Notwendig sind echte Optionen der tatsächlichen Überwindung der Sektorengrenzen, weiter noch entbürokratisierte Verwaltungsprozesse und Erleichterung der sicheren Datenbänke etc. Zu bedenken ist die thematisch wie fachlich erforderliche Vernetzung in der Pflegebranche in jegliche anderen Branchen, da das Pflegeszenario alle Lebensbereiche intrinsisch berührt. Komplette Versorgungspfade, erst einmal „nur“ aus pflegerischer Sicht betrachtet, finden in mehreren unterschiedlichen „Sektoren“ statt (die in der Tat noch bestehen, auch wenn die Forderung der Überwindung der Sektoren und die nach übergreifenden Konzepten wieder lauter wird). Die Komplexität, die gelingende Pflege $360^{\circ}$ bedeutet und erfordert, ist nur unter höchsten Anforderungen an (Fach-)Wissen und (Sprach-)Kenntnissen zu beherrschen. Die Crux ist, dass das Bestreben der Reduktion von Komplexität gleichzeitig neue Komplexität hervorruft und das Gegenteil von Komplexität nicht Einfachheit ist (Bolz 2012, 35 u. 41). Unter Berücksichtigung dieser Erkenntnisse und gleichzeitig im Hinblick auf das, was Digitalisierung leisten soll, gelingen diese Neuerungen sicher erst recht nicht, wenn im Vorfeld schon mit unpräzisen Definitionen geplant wird.

\section{Dimensionen erforderlicher Perspektiven auf die Sprache}

e-Patient Dave, der Autor Dave deBronkart, hat aus der eigenen Erfahrung heraus Bücher geschrieben, in denen er dazu aufruft, wie Leistungserbringer besser in Netzen arbeiten können, damit es den Patienten zu Gute kommt. Er fordert „Lasst Patienten mithelfen!“ und regt an, ab sofort nicht mehr von Healthcare, sondern von health \& care zu sprechen. Er will ein anderes Bewusstsein unter denen, die den Versorgungsprozess gemeinsam gestalten. Er fordert, dass alle differenzierter über Gesundheit nachdenken müssen. Vor seiner Krebserkrankung arbeitete er im Marketing und als Mensch der Sprache formuliert er, sich der Macht der Sprache bewusst zu sein, setzt sie ein und erzielt Wirkung durch Präzision: Gesundheit ist nicht Medizin und Behandlung ist nicht zwingend mit Pflege und oder Fürsorge verbunden. Er sagt:

„Sprache bestimmt unser Denken, daher finde ich es gut, sich darüber im Klaren zu sein, worüber wir diskutieren. Lassen Sie uns die Begriffe, Medizin', ,Behandlung', ,Pflege und ,Fürsorge" klar und bewusst werden." (vgl. Bellinger u. Krieger 2014, 35) 
Abschließend sei das Plädoyer noch um das Erfordernis des Sinns bereichert. Sprache als Herausforderung für den gelingenden Digitalisierungsprozess zu verstehen ist das eine. Sprache aber darf nicht Selbstzweck sein, sondern sie muss immer mit Inhalten einhergehen, die Sinn in dem speziell relevanten Kontext ergeben. Der Kontext - hier die Pflege - gibt den inhaltlichen Rahmen, wie schon erwähnt. Dabei sei noch darauf hingewiesen, dass es „die Pflege“ nicht gibt. Es gibt eine Pflegebranche, unter deren Dach sich thematisch oberflächlich alles subsummieren lässt, was mit Sorgestrukturen, Kümmerung, Fürsorge und Pflege zu tun hat, auch das, was die Pflegeversicherung impliziert, aber diese reicht für die Gesamtheit dessen, was „Pflege“ ausmacht, nicht aus. „Die Pflege“ soll nicht in eines der zwangsbeschränkten „Kollektivsingularien“ eingereiht werden, wie „die Geschichte“, „die Wirklichkeit“ oder „der Mensch“, die es in Wahrheit nach Bolz nur als Vielzahl gibt und die sich folglich unterschiedlich ausprägen (Bolz 2012, 28). Die ist auf „die Pflege“, die als Kollektivsingular nicht existiert, übertragbar und bedarf unbedingt konsequent differenzierter Definitionen.

\section{Eine Art Fazit mit Aufruf zur bewussten Sprachkultur}

Chomsky weiß, dass die Sprache das Bemerkenswerteste unseres Selbst ist. Bolz sagt, dass Ideen die „Institutionen des Denkens“ sind (vgl. Bolz 2012, 29), Bellinger und Krieger kommen zur Erkenntnis, dass Sprache unser Denken bestimmt. So sind Ideen nur durch Sprache möglich und wie Bolz weiter sagt, brauchen „wir also neue klare Begriffe - aber in dem Bewusstsein, dass es sich um Hilfskonstruktionen handelt“. Dass Transformation und Entstehung von neuem immer dem Wandel unterliegen, ist Voraussetzung jeglicher (lebendiger) Entwicklung. Sprache aber muss zwingend als Trägerin und Generatorin sinnhafter Inhalte anerkannt werden, denn nur so gelingen Prozesse, wie auch die Digitalisierung, da Verständlichkeit und Eindeutigkeit in der Definition, Bedeutung und Zielausrichtung anders nicht hergestellt werden kann. Und in der Referenz auf unser Selbst, das sich auch durch die Sprache definiert, wird gleichsam auf die Verantwortung eines jeden reflektiert, mit Sprache bestenfalls sinnvoll und zielgerichtet umzugehen, diese einzusetzen und sich somit letztlich auch auf diese Weise aktiv an der gesamtgesellschaftlichen Aufgabe der pflegerischen Versorgung zu beteiligen, in dem Systemerweiterung mit Expertise und hohem Qualitätsanspruch gelingen kann.

\section{Literatur}

Bellinger A, Krieger DI (2014) Gesundheit 2.0. transcript Verlag, Bielefeld, 1.3.8 S. 34ff.

Bolz N (2012) Die Sinngesellschaft. Kulturverlag Kadmos, Berlin, S. $28 f f$.

Bundesministerium für Gesundheit (BMG) (2017) Mit Datenschutz zum Datenschatz - Was die Digitalisierung des Gesundheitswesens leisten muss. URL: http://www.bundesgesundheitsministerium.de/presse/interviews/interviews-2017/nambensbeitrag-faz.html\#c9839 (abgerufen am 01.05.2017)

Bundesministerium für Wirtschaft und Energie (BMWI) (o.J.) Den digitalen Wandel gestalten. URL: https:// www.bmwi.de/Redaktion/DE/Dossier/digitalisierung.html (abgerufen am 28.04.2017)

Chomsky N (2016) Was für Lebewesen sind wir? Suhrkamp Verlag, Berlin, S. 38

Gabler Wirtschaftslexikon (o.J.) Programmiersprache. URL: http://wirtschaftslexikon.gabler.de/Definition/programmiersprache.html (abgerufen am 23.04.2017)

Watzlawick P, Beavin JH, Jackson DD (1969) Menschliche Kommunikation. Huber, Bern, Stuttgart, Wien, S. 53 